\title{
The validation of the 2014 International Society of Urological Pathology (ISUP) grading system for patients with high-risk prostate cancer: a single- center retrospective study
}

This article was published in the following Dove Press journal:

Cancer Management and Research

\author{
Jiandong Liu' ${ }^{1} * *$ \\ Jinge Zhao',* \\ Mengni Zhang ${ }^{2}$ \\ $\mathrm{Ni}$ Chen $^{2}$ \\ Guangxi Sun' \\ Yaojing Yang' \\ Xingming Zhang' \\ Junru Chen' \\ Pengfei Shen' \\ Ming Shi ${ }^{1}$ \\ Hao Zeng' \\ 'Department of Urology, Institute of \\ Urology, West China Hospital, Sichuan \\ University, Chengdu 61004I, People's \\ Republic of China; ${ }^{2}$ Department of \\ Pathology, West China Hospital, Sichuan \\ University, Chengdu 61004I, People's \\ Republic of China
}

*These authors contributed equally to this work
Correspondence: Ming Shi; Hao Zeng West China Hospital, Sichuan University, Chengdu 61004I, People's Republic of China

Fax +86288542245 I

Email smcm2014@I63.com;

kucaizeng@163.com
Introduction: Since the new 2014 grading system was recommended by the International Society of Urological Pathology (ISUP), it has been validated in patients with localized prostate cancer (PCa) and it has shown excellent prognostic value. However, its predictive power in high-risk PCa remains unclear.

Methods: A total of 420 patients with high-risk PCa who underwent radical prostatectomy (RP) were included in this study. Biochemical recurrence-free survival (BRFS) was set as the endpoint.

Results: Biochemical recurrence occurred in 84/420 (20.0\%) patients at the end of follow-up. Compared to the three-tier grouping system, the five-tier grouping system could more effectively distinguish the BRFS of patients with higher predictive accuracy (C-index: 0.599 vs 0.646$)$. The BRFS of patients with grade group (GG) 1 and GG 2 was similar $(P=0.593)$. Also, the prognosis between those with GG 2 and GG 3 could be clearly distinguished $(P=0.001)$. However, the discrimination capacity between patients with GG 3 and GG 4 was limited $(P=0.681)$. When tertiary Gleason pattern (TGP5) and intraductal carcinoma of the prostate (IDC-P) were excluded, the HR value of the GG 4 group $v$ s the GG 3 group increased from 1.15 (95\% CI: $0.59-2.22)$ to 1.49 (95\% CI: $0.72-3.10)$ and 1.36 (95\% CI:0.65-2.83), respectively.

Conclusions: This study is the first to validate the new 2014 ISUP grading system in patients with high-risk PCa who underwent RP. The 2014 system could effectively classify patients into five groups with high predictive accuracy. Notably, the existence of TGP5 and IDC-P needs to be routinely reported in clinical practice, which could help to support the predictive value of the new grading system.

Keywords: biochemical recurrence-free survival, ISUP grading system, high-risk, prostate cancer, radical prostatectomy

\section{Introduction}

The Gleason grading system has been one of the most powerful prognostic factors in prostate cancer (PCa) since it was first proposed in 1966 by Donald Gleason. ${ }^{1}$ In the past several decades, to improve its predictive ability, the Gleason grading system has been revised and updated several times. ${ }^{2,3}$

In 2005, the International Society of Urological Pathology (ISUP) convened a group of experts to make the first major modifications to the Gleason grading system. $^{2}$ The latest amendment of Gleason grading system was performed at the 
2014 ISUP Consensus Conference. ${ }^{3}$ According to the newest ISUP criteria, a novel five-tiered set of Gleason grade groups (GGs) was established and recommended. Based on the new 2014 system, patients could be classified into GG 1 (GS 3+3), GG 2 (GS 3+4), GG 3 (GS 4+3), GG 4 (GS 8), and GG 5 (GS 9-10).

Subsequently, several studies validated the new ISUP grading system in patients with different stages of $\mathrm{PCa} .{ }^{4-12}$ Almost all of the validated studies among patients with localized PCa who underwent either radical prostatectomy (RP) or radical radiotherapy (RT) demonstrated the superiority of the new ISUP system. ${ }^{4-10}$ However, data from our medical center challenged the utilization of the new ISUP grading system in patients with metastatic PCa. ${ }^{12}$ Compared to low- or intermediate-risk localized $\mathrm{PCa}$, patients with high-risk $\mathrm{PCa}$ always had a higher probability of recurrence, even patients who received treatments with the potential for a complete cure. ${ }^{13}$ However, to date, the validation of the new 2014 ISUP grading system in patients with high-risk PCa has not been reported, and its predictive power in this subpopulation needs to be evaluated.

The aim of this study was to validate the predictive value of the 2014 new ISUP grading criteria in predicting biochemical recurrence in patients with high-risk $\mathrm{PCa}$ using specimens from RP.

\section{Materials and methods}

\section{Patient population}

According to the D'Amico et al criteria of high-risk nonmetastatic PCa (PSA $\geq 20 \mathrm{ng} / \mathrm{mL}$ or GS $\geq 8$ or T stage $\geq$ T2c), ${ }^{14}$ a total of 420 patients with high-risk PCa who underwent RP in our center between 2009 and 2015 were included and retrospectively analyzed in this study. Among these, 352/420 (83.8\%) underwent laparoscopic RP, while only 68/420 (16.2\%) underwent robot-assisted laparoscopic RP, mainly because robot-assisted operation was implemented late in China. A total of 206/420 $(49.0 \%)$ men were treated with adjuvant androgen-deprivation therapy (ADT) and/or adjuvant radiotherapy (RT) after surgery. The cut-off point for analysis was June 1, 2017. The median follow-up time was 40.5 months.

All of the surgical specimens and pathological grading patterns were retrieved and reviewed independently and separately by two urological pathologists (Ni Chen and Mengni Zhang) according to the 2014 ISUP grading criteria. $^{2,3}$ If disagreement arose, then a consensus was reached by further consultation with a third pathologist
(Zhou Qiao). Tertiary Gleason pattern 5 (TGP5) is assigned if the tertiary component constitutes $\leq 5 \%$ of an entire tumor that otherwise demonstrates a Gleason pattern of 3 or $4 .^{2,3}$ Pre- or post-operative clinicopathologic characteristics of all of the cases were collected, including GS, age, baseline serum prostate-specific antigen (PSA) level, pelvic lymph node dissection (PLND), pathological T stage, the presence of intraductal carcinoma of the prostate (IDC-P), extraprostatic extension (EPE) status, seminal vesicle invasion (SVI) status, and positive surgical margin (PSM) status. The presence of IDC-P was reported routinely in our center.

\section{End point definition}

Due to the relatively short follow-up time, biochemical recurrence-free survival (BRFS) instead of overall survival (OS) was chosen as the end point in the current study. Biochemical recurrence was defined as two consecutive increases in serum PSA $\geq 0.2 \mathrm{ng} / \mathrm{ml}$ after RP. ${ }^{15,16}$ BRFS was the period from RP to the time of biochemical recurrence.

\section{Statistical methods}

Continuous variables were presented as the median and interquartile range (IQR), while categorical variables were reported as the number and percentage. The chi-square test and the Mann-Whitney $U$ test were used to compare the baseline characteristics between patients with various GS values. Kaplan-Meier curves were used to compare the BRFS according to either the three-tier or five-tier grouping system. Differences in BRFS between each group were compared using the log-rank test. Univariate and multivariate analyses were conducted by Cox regression model as follows. First, the univariate Cox regression was used to evaluate the ability of each variable to predict BRFS. Second, parameters with $P<0.05$ in the univariate analysis were further analyzed by multivariate Cox regression. The accuracy and discrimination ability of the 2014 ISUP grading criteria according to the three-tier and five-tier grouping systems were evaluated and compared using the concordance index (C-index).

Data analyses were performed using SPSS software (version 21.0). All of the tests were two-sided. A $p$-value below 0.05 was considered to be statistically significant.

\section{Results}

\section{Patient characteristics}

The clinicopathological characteristics of the whole cohort are shown in Table 1. According to the three-tier grouping system, 25 (6.0\%), 268 (63.8\%), and 127 (30.2\%) men were 


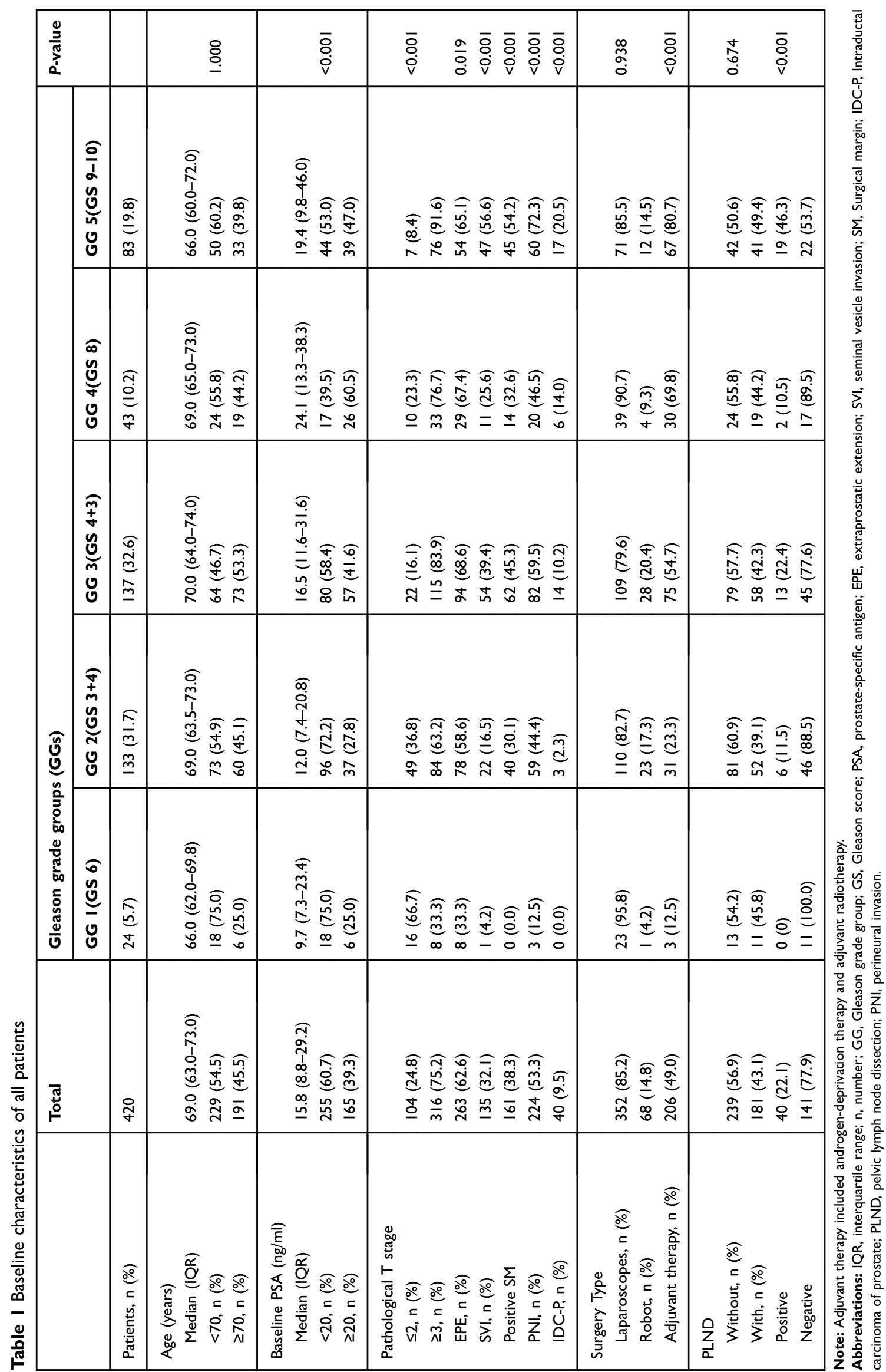


GS 6, 7, and 8-10, respectively. While according to the fivetier grouping system, 24 (5.7\%), 133 (31.7\%), 137 (32.6\%), 43 (10.2\%), and 83 (19.8\%) were GG 1 (GS 6), GG 2 (GS 3 +4), GG 3 (GS 4+3), GG 4 (GS 8), and GG 5 (GS 9-10), respectively. The proportions of EPE, SVI, PSM, and IDC-P in total patients were $62.6 \%, 32.1 \%, 38.3 \%$, and $9.5 \%$, respectively. The higher Gleason grade groups were associated with a higher baseline PSA level, higher pathological T stage, larger percentage of EPE, SVI, higher presence of IDC-P, and higher frequency of lymph node metastasis. The median age was 69 years. At the end of the follow-up, biochemical recurrence occurred in 84/420 (20.0\%) patients.

\section{Comparison of the predictive value of the five-tier and the three-tier Gleason grading grouping system}

The prognostic predictive value of the three-tier grouping and the five-tiered grouping system according to 2014 ISUP criteria was compared and visually presented by the Kaplan-
Meier curves (Figure 1). Based on the three-tier grouping, the BRFS of patients with GS 6, GS 7, and GS 8-10 could be distinguished (5-year BRFS probability: 0.89, 0.75, and 0.54, $p<0.001$, Figure 1A). According to the five-tier system, the 5year BRFS probabilities of GG $1-5$ were $0.89,0.87,0.62$, 0.60 , and 0.50 , respectively ( $p<0.001$, Figure $1 \mathrm{~B})$. The predictive accuracy evaluation showed that the predictive value of the five-tiered Gleason grade groups according to 2014 ISUP criteria was superior to that of the three-tier grouping (C-index: 0.646 vs 0.599). Of note, more detailed information among patients with different GS groups was revealed by the five-tier criteria. In the five-tier 2014 ISUP criteria, the BRFS of patients with GG 1 and GG 2 was very similar $(P=0.593)$, and the prognosis of GG 2 and GG 3 could be clearly distinguished $(P=0.001)$. However, the discrimination capacity between patients with GG 3 and GG 4 was relatively limited $(P=0.681)$. GG 5 clearly exhibited the worst prognosis among the whole cohort (mBRFS of GG4 vs mBRFS of GG5: $P=0.029)$.
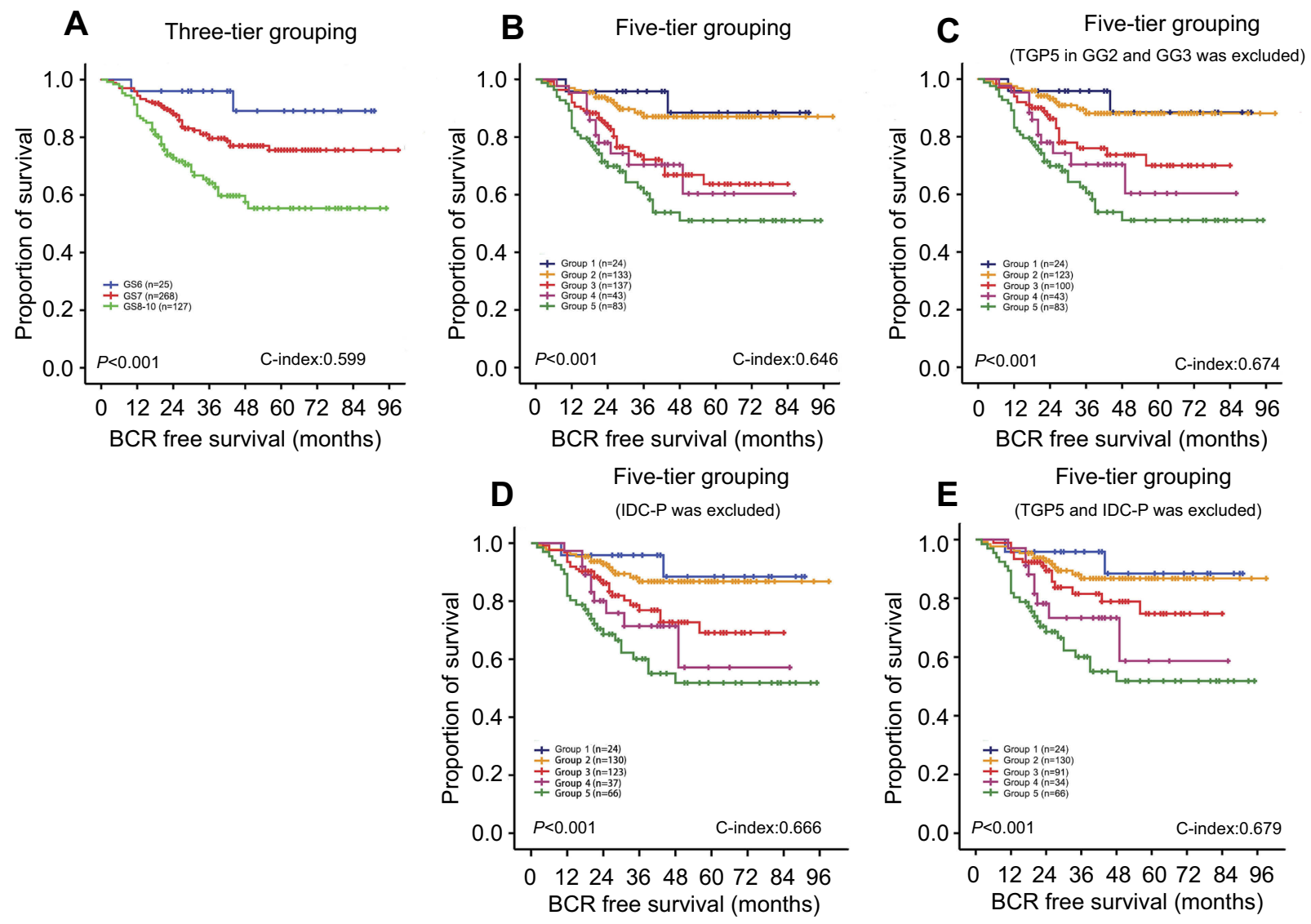

Figure I Kaplan-Meier curves of BRFS. (A) BRFS curves according to the three-tier grouping system; (B) BRFS curves according to the five-tier grouping system; (C) BRFS curves with the five-tier grouping system with GG3 patients with the tertiary GS 5 excluded; (D) BRFS curves with the five-tier grouping system with IDC-P excluded; (E) BRFS curves with the five-tier grouping system with TGP5 and IDC-P excluded.

Abbreviations: BCR, biochemical recurrence; BRFS, biochemical recurrence-free survival; GG, Gleason grade group; GS, Gleason score; IDC-P, intraductal carcinoma of the prostate; TGP5, tertiary Gleason pattern. 


\section{Univariate and multivariate analyses of BRFS in high-risk PCa}

The univariate and multivariate analyses of BRFS were conducted using the Cox proportional model (Table 2). Since that only 24 (5.7\%) patients were in GG 1 and that patients in GG 2 had very similar prognoses to those in GG 1 (HR: $1.50,95 \%$ CI: 0.34-6.59, $P=0.595$ ), GG 1 combined with GG 2 was set as the reference in the Cox regression analyses of the five-tier 2014 ISUP grading system. Univariate analyses indicated that both the threetier and the five-tier grouping systems, baseline PSA, pathological T stage, IDC-P, EPE, SVI, and PSM status were all prognosticators of BRFS. In the multivariate analyses, in addition to the five-tier GS score, the baseline PSA, EPE, and SVI were also independent predictors of BRFS in patients with high-risk PCa after RP. The univariate and multivariate analyses were also conducted in 181 patients who underwent PLND. The univariate analyses of cases with PLND indicated that positive lymph node was a prognosticator of BRFS $(\mathrm{HR}=2.03,95 \% \mathrm{CI}$ :
1.033-3.991, $P=0.040)$. However, multivariate analyses revealed that IDC-P and the five-tier 2014 ISUP grading system were the only two prognosticators of BRFS, whereas positive lymph node was not an independent predictor of BRFS (HR=1.154, 95\%CI: 0.533-2.498, $\mathrm{P}=0.717$ ).

\section{The impact of TGP5 and IDC-P on the predictive accuracy of the 2014 ISUP criteria in high-risk $\mathrm{PCa}$}

A subset of patients had TGP5 as a comment in their pathological reports. Despite its low proportion in the whole tumor burden, TGP5 might impact the prognosis prediction of the five-tier 2014 ISUP grading system due to its aggressive biological character. Survival analysis was re-conducted excluding the men with TGP5 in GG 3 (Figure 1C). In the whole cohort, the presence of TGP5 was reported and confirmed in 52/420 (12.4\%) men with high-risk PCa. Among them, 37/137(27.0\%) cases were GG 3, 10/133 (7.5\%) were GG 2, and only 5/43 (11.6\%)

Table 2 Univariate and multivariate analyses of BRFS

\begin{tabular}{|c|c|c|c|c|}
\hline & \multicolumn{2}{|c|}{ Univariate analyses } & \multicolumn{2}{|c|}{ Multivariate analyses } \\
\hline & HR (95\%Cl) & $p$-value & HR (95\%Cl) & $p$-value \\
\hline \multicolumn{5}{|l|}{ Baseline PSA (ng/ml) } \\
\hline$>20$ vs $\leq 20$ & $1.94(|.30-2.9|)$ & 0.001 & $1.75(1.15-2.67)$ & 0.009 \\
\hline \multicolumn{5}{|l|}{ Pathological T stage } \\
\hline$\geq 3$ vs $\leq 2$ & $2.5 \mathrm{I}(\mathrm{I} .40-4.5 \mathrm{I})$ & 0.002 & & \\
\hline \multicolumn{5}{|l|}{ EPE } \\
\hline With vs Without & $1.95(1.21-3.15)$ & 0.006 & $2.49(|| 7-5.31)$. & 0.018 \\
\hline \multicolumn{5}{|l|}{ SVI } \\
\hline With vs Without & $2.4 \mathrm{I}(\mathrm{I} .6 \mathrm{I}-3.60)$ & 0.000 & $1.41(0.88-2.25)$ & 0.154 \\
\hline \multicolumn{5}{|l|}{ Positive SM } \\
\hline With vs Without & $1.70(1.12-2.57)$ & 0.012 & $1.12(0.72-1.74)$ & 0.615 \\
\hline \multicolumn{5}{|l|}{ IDC-P } \\
\hline With vs Without & $2.49(I .47-4.2 I)$ & 0.001 & $1.56(0.88-2.74)$ & 0.125 \\
\hline \multicolumn{5}{|l|}{ Three-tier grading criteria } \\
\hline 7 vs 6 & $2.88(0.70-11.85)$ & 0.143 & & \\
\hline $8-10$ vs 6 & $6.29(1.52-25.94)$ & 0.011 & & \\
\hline \multicolumn{5}{|l|}{ Five-tier grading criteria (GGs) } \\
\hline GG 3 vs GG I or GG 2 & $2.91(1.60-5.28)$ & 0.000 & $2.11(1.14-3.91)$ & 0.017 \\
\hline GG 4 vs GG I or GG 2 & $3.29(1.55-6.96)$ & 0.002 & $2.49(1.16-5.34)$ & 0.019 \\
\hline GG 5 vs GG I or GG 2 & $4.89(2.69-8.88)$ & 0.000 & $3.18(1.68-6.02)$ & 0.000 \\
\hline
\end{tabular}

Note: Adjuvant therapy included androgen-deprivation therapy and adjuvant radiotherapy.

Abbreviations: BRFS, biochemical-recurrence free survival; GG, Gleason grade group; HR, hazard ratio; Cl, confidence interval; PSA, prostate-specific antigen; EPE, extraprostatic extension; SVI, seminal vesicle invasion; SM, surgical margin; IDC-P, intraductal carcinoma of prostate; ISUP, International Society of Urological Pathology. 
were GG 4. In addition, in patients in the GG 3 group, TGP5 was strongly associated with a higher baseline PSA level $(P=0.010)$, higher pathological $\mathrm{T}$ stage $(P=0.039)$, and larger percentage of SVI $(P=0.082)$ (Table 3$)$. Additionally, the patients in GG 3 group with TGP5 were more likely to progress to biochemical recurrence than those without TGP5 $(\mathrm{HR}=2.05,95 \% \mathrm{CI}$ : $1.03-4.11$, $P=0.043)$. The comparison of clinicopathologic parameters between patients of GG 2 with or without tertiary GS 5 is shown in Table 4.

Interestingly, the Kaplan-Meier curves indicated that, without the interference of TGP5, the prognosis discrimination between GG 3 and GG 4 group was numerically greater (C-index: 0.674) (Figure 1C). Although still without statistical significance, the HR value of the GG 4 group $v s$ the GG 3 group increased from $1.15(95 \% \mathrm{CI}$ : $0.59-2.22$ ) to 1.49 (95\%CI: $0.72-3.10)$.

Since IDC-P has been proven to be associated with poor prognosis and was recognized as a newly pathological entity in the 2016 WHO classification, we did the same analysis for IDC-P as TGP5 and obtained similar results (C-index: 0.666) (Figure 1D), the HR value of the GG 4 group $v s$ the GG 3 group increased to 1.36 (95\%CI:0.652.83). Furthermore, when TGP5 and IDC-P were both excluded, prognosis discrimination between $\mathrm{GG} 3$ and GG 4 groups further increased (C-index: 0.679) (Figure $1 \mathrm{E})$, and the HR value of the GG 4 group $v s$ the GG 3 group increased to HR 1.79 (95\%CI: 0.78-4.11).

\section{Discussion}

In 2014, the ISUP consensus conference gathered over 80 experts, including pathologists, urologists, radiation oncologists, and medical oncologists, to amend and update the old GS grading system. The main reasons for this updating were that several grading issues were unsolved or not covered in the 2005 criteria, new pertinent studies had emerged, and the clinical practice in PCa had changed. ${ }^{3}$

In the past three years, several centers have attempted to validate the clinical significance of the new 2014 ISUP grading system. ${ }^{4-12}$ However, most of these works were based on populations with localized (low- to intermediaterisk) $\mathrm{PCa}$, and almost all of the validations showed the superiority of the new 2014 criteria. As we all know, with the disease progression and the heterogeneity of PCa, the prognosis of the disease could be impacted not only by pathological grading but also by other miscellaneous clinical-pathological risk factors. In one of our previous studies, we found that the predictive accuracy of the three-tier grouping system seemed not to be inferior to that of the five-tier grouping system in patients initially diagnosed with metastatic PCa. The biological characteristics of high-risk PCa are quite different from those of localized $\mathrm{PCa}$. Due to its higher risk of biochemical/clinical recurrence, the ability of clinicians to evaluate and predict the prognostic probability accurately is more important; however, to date, the new 2014 ISUP grading criteria has not been exclusively validated among this sub-population.

To the best of our knowledge, in the current study, we conducted the first validation of the 2014 ISUP grading system in patients with high-risk PCa who underwent RP. Compared to the three-tier platform, the five-tier grouping system showed excellent ability with higher predictive accuracy among patients with high-risk PCa.

Table 3 The comparison of clinicopathologic parameters between patients of GS 4+3 with or without tertiary GS 5

\begin{tabular}{|l|l|l|l|l|}
\hline & GS 4+3 (total) & GS 4+3 (with tertiary GS 5) & GS 4+3 (without tertiary GS 5) & P-value \\
\hline Number & 137 & 37 & 100 & \\
\hline $\begin{array}{l}\text { Baseline PSA (ng/ml) } \\
<20, \mathrm{n}(\%)\end{array}$ & $80(58.4)$ & $15(40.5)$ & $65(65.0)$ & $35(35.0)$ \\
$\geq 20, \mathrm{n}(\%)$ & $57(41.6)$ & $22(59.5)$ & & 0.010 \\
\hline Pathological T stage & & & $20(20.0)$ & $80(80.0)$ \\
$\leq 2, \mathrm{n}(\%)$ & $22(16.1)$ & $2(5.4)$ & $68(68.0)$ & $35(35.0)$ \\
$\geq 3, \mathrm{n}(\%)$ & $115(83.9)$ & $35(94.6)$ & $44(44.0)$ & 0.039 \\
EPE (\%), n (\%) & $94(68.6)$ & $26(70.3)$ & $9(9.0)$ & 0.799 \\
SVI (\%), n (\%) & $54(39.4)$ & $19(51.4)$ & $18(48.6)$ & 0.082 \\
Positive SM, n (\%) & $62(45.3)$ & $5(13.5)$ & 0.439 \\
IDC-P, n (\%) & $14(10.2)$ & & \\
\hline
\end{tabular}

Abbreviations: GS, Gleason score; PSA, prostate-specific antigen; EPE, extraprostatic extension; SVI, seminal vesicle invasion; SM, surgical margin; IDC-P, intraductal carcinoma of prostate. 
Table 4 The comparison of clinicopathologic parameters between patients of GS $3+4$ with or without tertiary GS 5

\begin{tabular}{|c|c|c|c|c|}
\hline & GS $3+4$ (total) & GS $3+4$ (with tertiary GS 5) & GS $3+4$ (without tertiary GS 5) & $P$-value \\
\hline Number & 133 & 10 & 123 & \\
\hline \multicolumn{5}{|l|}{ Baseline PSA (ng/ml) } \\
\hline$<20, \mathrm{n}(\%)$ & $96(72.2)$ & $6(60.0)$ & $90(73.2)$ & 0.371 \\
\hline$\geq 20, \mathrm{n}(\%)$ & $37(27.8)$ & $4(40.0)$ & $33(26.8)$ & \\
\hline \multicolumn{5}{|l|}{ Pathological T stage } \\
\hline$\leq 2, \mathrm{n}(\%)$ & $49(36.8)$ & $2(20.0)$ & $47(38.2)$ & 0.251 \\
\hline$\geq 3, \mathrm{n}(\%)$ & $84(63.2)$ & $8(80.0)$ & $76(61.8)$ & \\
\hline EPE (\%), n (\%) & $78(58.6)$ & $8(80.0)$ & $70(56.9)$ & 0.154 \\
\hline SVI (\%), n (\%) & $22(16.5)$ & $2(20.0)$ & $20(16.3)$ & 0.760 \\
\hline Positive SM, n (\%) & $44(33.1)$ & $4(40.0)$ & $40(32.5)$ & 0.629 \\
\hline IDC-P, n (\%) & $3(2.3)$ & $0(0.0)$ & $3(2.4)$ & 0.617 \\
\hline
\end{tabular}

Abbreviatins: GS, Gleason score; PSA, prostate-specific antigen; EPE, extraprostatic extension; SVI, seminal vesicle invasion; SM, surgical margin; IDC-P, intraductal carcinoma of prostate.

The most important value of a grading system is to predict the prognosis and help to guide treatment decisions. Mounting evidence has proven that the three-tier grouping using a simplified single GS of 7 failed to detect differences in prognosis between GS $3+4$ and GS $4+3 .{ }^{17-20}$ Undoubtedly, the greatest superiority of the new grading system is its discrimination between GG 2 (GS 3+4) and GG 3 (GS 4+3), which should be of importance in preventing some patients with GS7 (GG 2) from overtreatment.

However, more attention should be paid to the fact that, in contrast to its performance in patients with localized $\mathrm{PCa}$, there were still some defects in the new 2014 ISUP system among the high-risk sub-population. The BRFS of men with GG 3 (GS 4+3) and GG 4 (GS 8) was difficult to distinguish. A similar phenomenon was also observed in several previous validation studies. ${ }^{4-7}$ Epstein et al considered that one reasonable explanation might be that more patients with GG 4 than with GG 3 received hormone therapy, which could neutralize the higher aggressive ability of GG $4 .^{4}$

In the present study, a higher proportion of patients with GG 4 than with GG 3 received adjuvant therapy after surgery $(54.7 \%$ vs $69.8 \%)$, but the difference was not statistically significant $(P=0.081)$. We also observed that among 137 patients with GG 3, pathologists reported TGP5 in $27 \%$ (37/137). The higher occurrence of TGP5 might be considered a contaminating factor, which could explain the overlapping of the survival curves of GG 3 and GG 4.

Sub-analysis among the GG 3 group subsequently demonstrated that patients with TGP5 had more aggressive characteristics than those without, in terms of baseline
PSA levels, pathological $\mathrm{T}$ stage, and the percentage of SVI. TGP5 was also observed to increase the odds of biochemical recurrence in the GG 3 patients. The prognostic value of TGP5 remains controversial. ${ }^{21-24}$ Several studies have proposed that GS $4+3$ patients and TGP5 who underwent RP should be considered as GG 3 patients with a minor higher grade component. ${ }^{22-24}$ All of these clues hinted that the existence of TGP5 in GG 3 patients plays a crucial role in accounting for the overlap of BRFS between GG 3 and GG 4. In fact, when patients with GG3 concomitant with TGP5 were excluded from the sub-analyses in our study, the difference in BRFS between the GG 4 and GG 3 groups improved (HR from 1.15 to 1.49). At the same time, the presence of cribriform cancer or IDC-P might play a role similar to that of TGP5. A series of studies confirmed that cribriform cancer or IDC-P was associated with bad prognosis for localized $\mathrm{PCa}$ patients treated with RP or radiotherapy. ${ }^{25}$ When patient with IDC-P was excluded, the difference in BRFS between the GG 4 and GG 3 groups improved (HR from 1.15 to 1.36). Since cribriform cancer was not reported specifically in our center, it was not analyzed in this study. Thus, both urological pathologists and urologists should pay more attention to the existence of TGP5 and IDC-P.

Furthermore, multivariate analyses showed that apart from the GS grading system, other clinicopathological parameters, including baseline PSA and EPE status, were also independent prognosticators for biochemical recurrence among patients with high-risk PCa. These confounding factors might also interfere with the prognostic prediction ability of the new ISUP grading system. In 
fact, in clinical practice, prognostic prediction for patients with high-risk prostate cancer should be comprehensively evaluated and thoroughly considered.

Several limitations existed in this study. First, this is a retrospective study with defects associated with its study type. Second, all of the data were based on a cohort of patients treated in a single center, and thus, biases such as treatment selection are unavoidable. Third, since there are currently no uniform criteria for the tertiary patterns in the GS grading system, our definition might not be completely in accordance with others. The main limitation of the current study was that, due to the relatively short followup time, BRFS was set as the end point instead of OS or cancer-specific survival. This limitation was unavoidable because the modified Gleason system was first introduced in 2005. Another 10-15 years must pass before the followup time is sufficient to use death as the end point. ${ }^{4}$

\section{Conclusion}

To our knowledge, this study is the first to validate the new 2014 ISUP grading system in patients with high-risk PCa who underwent RP. Despite miscellaneous risk factors, the five-tier 2014 grading system was still considered as an independent prognostic risk factor with high predictive accuracy. The presence of TGP5 and IDC-P showed major impact on the prognosis of patients with high-risk localized prostate cancer. Hence, in the clinic, the prognostic prediction for patients with high-risk PCa should be comprehensively evaluated and thoroughly considered. In addition, more attention should be paid to the existence of the TGP5 and IDC-P, and it should be routinely reported by pathologists in clinical practice to maintain the predictive ability of the new 2014 ISUP grading system.

\section{Ethics}

The study was approved by the Medical Ethics Committee of Sichuan University. All patients signed informed consent before undergoing RP.

\section{Acknowledgments}

This work was supported by grants from the National Natural Science Foundation of China (NSFC; 81402110, 81672547, 81572380, and 81272820), the Science and Technology Support Program of Sichuan Province (2015SZ0142), and the 1.3.5 Project for Disciplines of Excellence, West China Hospital, Sichuan University.

An abstract of this paper was presented at the following two conferences as posters: 1) 43rd ESMO Congress;
2) The 2019 Genitourinary Cancers Symposium. The posters' abstracts were published in "Poster Abstracts" in Annals of Oncology and Journal of Clinical Oncology.

\section{Author contributions}

All authors contributed towards data analysis, drafting and critically revising the paper, gave final approval of the version to be published, and agreed to be accountable for all aspects of the work.

\section{Disclosure}

The authors report no conflicts of interest in regard to this work.

\section{References}

1. Bailar JR, Mellinger GT, Gleason DF. Survival rates of patients with prostatic cancer, tumor stage, and differentiation-preliminary report. Cancer Chemother Rep. 1966;50:129-136.

2. Epstein JI, Allsbrook WJ, Amin MB, et al. The 2005 International Society Of Urological Pathology (ISUP) Consensus Conference on Gleason grading of prostatic carcinoma. Am J Surg Pathol. 2005;29:1228-1242.

3. Epstein JI, Egevad L, Amin MB, et al. The 2014 International Society of Urological Pathology (ISUP) Consensus Conference on Gleason grading of prostatic carcinoma: definition of grading patterns and proposal for a new grading system. Am J Surg Pathol. 2016;40:244-252. doi:10.1097/PAS.0000000000000530

4. Epstein JI, Zelefsky MJ, Sjoberg DD, et al. A contemporary prostate cancer grading system: a validated alternative to the Gleason score. Eur Urol. 2016;69:428-435. doi:10.1016/j.eururo.2015.06.046

5. Loeb S, Folkvaljon Y, Robinson D, et al. Evaluation of the 2015 Gleason grade groups in a nationwide population-based cohort. Eur Urol. 2016;69:1135-1141. doi:10.1016/j.eururo.2015.11.036

6. Samaratunga H, Delahunt B, Gianduzzo T, et al. The prognostic significance of the 2014 International Society of Urological Pathology (ISUP) grading system for prostate cancer. Pathol $J$ RCPA. 2015;47:515-519.

7. Delahunt B, Egevad L, Srigley JR, et al. Validation of International Society of Urological Pathology (ISUP) grading for prostatic adenocarcinoma in thin core biopsies using TROG 03.04 'RADAR'trial clinical data. Pathol J RCPA. 2015;47:520-525.

8. Rusthoven CG, Waxweiler TV, DeWitt PE, et al. Gleason stratifications prognostic for survival in men receiving definitive external beam radiation therapy for localized prostate cancer. Urol Oncol. Elsevier, 2015;33:71. e11-e71. e19. doi:10.1016/j.urolonc.2014.07.010

9. Spratt DE, Cole AI, Palapattu GS, et al. Independent surgical validation of the new prostate cancer grade-grouping system. BJU Int. 2016;118:763-769. doi:10.1111/bju.13488

10. Ham WS, Chalfin HJ, Feng Z, et al. New prostate cancer grading system predicts long-term survival following surgery for Gleason score 8-10 prostate cancer. Eur Urol. 2017;71:907-912. doi:10.1016/j.eururo.2016.11.006

11. Dell'Oglio P, Karnes RJ, Gandaglia G, et al. The new prostate cancer grading system does not improve prediction of clinical recurrence after radical prostatectomy: results of a large, two-center validation study. Prostate. 2017;77:263-273. doi:10.1002/pros.23265

12. Sun GX, Shen PF, Zhang XM, et al. Predictive efficacy of the 2014 International Society of Urological Pathology Gleason grading system in initially diagnosed metastatic prostate cancer. Asian J Androl. 2017;19:57. 
13. D'amico AV, Moul J, Carroll PR, et al. Cancer-specific mortality after surgery or radiation for patients with clinically localized prostate cancer managed during the prostate-specific antigen era. J Clin Oncol. 2003;21:2163-2172. doi:10.1200/JCO.2003.01.075

14. D' Amico AV, Whittington R, Malkowicz SB, et al. Biochemical outcome after radical prostatectomy, external beam radiation therapy, or interstitial radiation therapy for clinically localized prostate cancer JAMA. 1998;280:969-974. doi:10.1001/jama.280.11.969

15. Stephenson AJ, Kattan MW, Eastham JA, et al. Defining biochemical recurrence of prostate cancer after radical prostatectomy: a proposal for a standardized definition. J Clin Oncol. 2006;24:3973-3978. doi:10.1200/JCO.2005.04.0756

16. Cookson MS, Aus G, Burnett AL, et al. Variation in the definition of biochemical recurrence in patients treated for localized prostate cancer: the American Urological Association Prostate Guidelines for localized prostate cancer update panel report and recommendations for a standard in the reporting of surgical outcomes. $J$ Urol. 2007;177:540-545. doi:10.1016/j.juro.2006.10.097

17. Chan TY, Partin AW, Walsh PC, et al. Prognostic significance of Gleason score 3+ 4 versus Gleason score 4+ 3 tumor at radical prostatectomy. Urology. 2000;56:823-827.

18. Merrick GS, Butler WM, Galbreath RW, et al. Biochemical outcome for hormone-naive patients with Gleason score $3+4$ versus $4+3$ prostate cancer undergoing permanent prostate brachytherapy. Urology. 2002;60:98-103.
19. Stark JR, Perner S, Stampfer MJ, et al. Gleason score and lethal prostate cancer: does 3+4=4+3 J Clin Oncol. 2009;27:3459-3464. doi:10.1200/JCO.2008.20.4669

20. Wright JL, Salinas CA, Lin DW, et al. Prostate cancer specific mortality and Gleason 7 disease differences in prostate cancer outcomes between cases with Gleason 4+ 3 and Gleason 3+ 4 tumors in a population based cohort. J Urol. 2009;182:2702-2707. doi:10.1016/j.juro.2009.08.026

21. Isbarn H, Ahyai SA, Chun FKH, et al. Prevalence of a tertiary Gleason grade and its impact on adverse histopathologic parameters in a contemporary radical prostatectomy series. Eur Urol. 2009;55:394-403. doi:10.1016/j.eururo.2008.08.015

22. Egevad L, Granfors T, Karlberg L, et al. Percent Gleason grade 4/5 as prognostic factor in prostate cancer diagnosed at transurethral resection. J Urol. 2002;168:509-513.

23. Jang WS, Yoon CY, Kim MS, et al. The prognostic role of tertiary Gleason pattern 5 in a contemporary grading system for prostate cancer. Prostate Cancer Prostatic Dis. 2017;20(1):93. doi:10.1038/pcan.2016.55

24. Baras AS, Nelson JB, Han M, et al. The effect of limited (tertiary) Gleason pattern 5 on the new prostate cancer grade groups. Hum Pathol. 2017;63:27-32. doi:10.1016/j.humpath.2016.12.008

25. Iczkowski KA, Paner GP, Van der Kwast T. The new realization about cribriform prostate cancer. Adv Anat Pathol. 2018;25:31-37. doi:10.1097/PAP.0000000000000168

\section{Publish your work in this journal}

Cancer Management and Research is an international, peer-reviewed open access journal focusing on cancer research and the optimal use of preventative and integrated treatment interventions to achieve improved outcomes, enhanced survival and quality of life for the cancer patient
The manuscript management system is completely online and includes a very quick and fair peer-review system, which is all easy to use. Visit http://www.dovepress.com/testimonials.php to read real quotes from published authors. 